\title{
Reusing the Optimized Code for JavaScript Ahead-of-Time Compilation
}

\author{
HYUKWOO PARK, SUNGKOOK KIM, JUNG-GEUN PARK, and SOO-MOOK MOON, \\ Seoul National University
}

\begin{abstract}
As web pages and web apps increasingly include heavy JavaScript code, JavaScript performance has been a critical issue. Modern JavaScript engines achieve a remarkable performance by employing tiered-execution architecture based on interpreter, baseline just-in-time compiler (JITC), and optimizing JITC. Unfortunately, they suffer from a substantial compilation overhead, which can take more than $50 \%$ of the whole running time. A simple idea to reduce the compilation overhead is ahead-of-time compilation (AOTC), which reuses the code generated in the previous run. In fact, existing studies that reuse the bytecode generated by the interpreter or the machine code generated by the baseline JITC have shown tangible performance benefits $[12,31,41]$. However, there has been no study to reuse the machine code generated by the optimizing JITC, which heavily uses profile-based optimizations, thus not easily reusable. We propose a novel AOTC that can reuse the optimized machine code for high-performance JavaScript engines. Unlike previous AOTCs, we need to resolve a few challenging issues related to reusing profile-based optimized code and relocating dynamic addresses. Our AOTC improves the performance of a commercial JavaScript engine by 6.36 times $(\max )$ and 1.99 times (average) for Octane benchmarks, by reducing the compilation overhead and by running the optimized code from the first invocation of functions. It also improves the loading time of six web apps by 1.28 times, on average.
\end{abstract}

\section{CCS Concepts: • Software and its engineering $\rightarrow$ Compilers; Scripting languages;}

Additional Key Words and Phrases: JavaScript, ahead-of-time compilation, optimizing just-in-time compilation, web app, JavaScriptCore engine

\section{ACM Reference format:}

Hyukwoo Park, Sungkook Kim, Jung-Geun Park, and Soo-Mook Moon. 2018. Reusing the Optimized Code for JavaScript Ahead-of-Time Compilation. ACM Trans. Archit. Code Optim. 15, 4, Article 54 (December 2018), 20 pages.

https://doi.org/10.1145/3291056

\footnotetext{
This work was supported by Basic Science Research Program through the National Research Foundation (NRF) of Korea funded by the Ministry of Science, ICT \& Future Planning (NRF-2017R1A2B2005562).

The preliminary idea of this article was proposed as a two-paged work-in-progress paper [32], but most parts of this article are new and original.

Authors' addresses: H. Park, S. Kim, J.-G. Park, and S.-M. Moon. Department of Electrical and Computer Engineering. Seoul National University, Seoul, Korea; emails: \{clover2123, skkeem, erebus, smoon\}@altair.snu.ac.kr.

Permission to make digital or hard copies of all or part of this work for personal or classroom use is granted without fee provided that copies are not made or distributed for profit or commercial advantage and that copies bear this notice and the full citation on the first page. Copyrights for components of this work owned by others than the author(s) must be honored. Abstracting with credit is permitted. To copy otherwise, or republish, to post on servers or to redistribute to lists, requires prior specific permission and/or a fee. Request permissions from permissions@acm.org.

(C) 2018 Copyright held by the owner/author(s). Publication rights licensed to ACM.

1544-3566/2018/12-ART54

https://doi.org/10.1145/3291056
} 


\section{INTRODUCTION}

JavaScript is a standard web programming language, along with HTML and CSS. Traditionally, JavaScript has been used as lightweight scripting for web pages, especially for interacting with the user inputs and modifying the web pages at the client side. However, as the web has evolved with new standards and libraries, such as HTML5 [16], WebGL [24], ECMAScript6 [7], JavaScript is increasingly undertaking complex computations, and even running in the server environment [28]. So, JavaScript performance has been a real issue, and the just-in-time compiler (JITC) that translates JavaScript code to machine code at run time has been popularly used [8].

Modern JavaScript engines used in WebKit [2], Chrome [9], and Firefox [25] browsers extend JITC by employing a tiered execution architecture based on adaptive compilation, which recompiles JavaScript code differently based on the "hotness" of a function. That is, when a JavaScript function is first called, it is parsed to an intermediate code, e.g., bytecode, which is then executed by the interpreter. When the function is found to be warm, the baseline JITC compiles the bytecode into basic machine code for better performance. If the function is executed more frequently, thus being hot, the optimizing JITC re-compiles it into highly-optimized code. In this way, JavaScript engines can trade-off the startup delay and the steady-state performance.

Although JavaScript performance has soared using tiered compilation, we found that the compilation overhead is extremely high. Figure 1 shows the overhead for each compilation tier of the WebKit JavaScriptCore (JSC) engine, measured for Octane benchmarks [11]. The total compilation overhead accounts for $52 \%$ of the whole JavaScript execution time, most of which is due to parsing and optimizing JITC. That is, parsing translates every invoked JavaScript function to the bytecode with lexical and syntactic analysis, while optimizing JITC performs many optimizations, especially profile-based optimizations. On the other hand, baseline JITC simply maps each bytecode to the corresponding machine code with little optimization, so the overhead is relatively low. For web apps, we could observe a lower yet still substantial (20\%) compilation overhead during app loading time, although there are far fewer hot functions that can enable the optimizing JITC (when we run the app in an event-driven manner after app loading, the overhead would be amortized and reduced, if the same event handlers and functions are executed repetitively).

To reduce the optimizing JITC overhead, concurrent compilation has been proposed [4, 13, 23], where optimizing JITC works on a separate thread, concurrently with the main thread to hide the compilation overhead. However, concurrent compilation requires multi-core CPUs, thus not generally applicable, and even with the multi-core CPUs, it cannot hide the interpretation overhead. Also some browsers (e.g., WebKit) enable concurrent compilation only for 64-bit operating system (OS) due to the inner value representation.

A simpler approach to reduce the compilation overhead is ahead-of-time compilation (AOTC), which saves the compiled code generated in the previous run and reuses it in the current run without compilation. Actually, AOTC has been successfully used for Java such as reusing the fully optimized JITC code [19, 20,39] or using the high-performance code obtained from bytecode-to-C $[21,29]$. AOTC for Java is relatively straightforward since its language features are static, allowing simple translation and reuse (allowing even hardware-based analysis and translation for Java) $[33,34,35]$. However, AOTC for JavaScript is much more complicated than Java, since its highly dynamic language features such as dynamic types, closures, or runtime addition/deletion of object properties, require more complex profile-based optimizations than Java; such optimized code is hard to generate and reuse, as will be explained shortly. In fact, previous AOTCs for JavaScript reuse only the bytecode generated by the interpreter or the machine code generated by the baseline JITC $[12,31,41]$.

This article proposes a novel AOTC approach for reusing the machine code generated by the optimizing JITC for the state-of-the-art JavaScript engines. It can obviate all compilation 


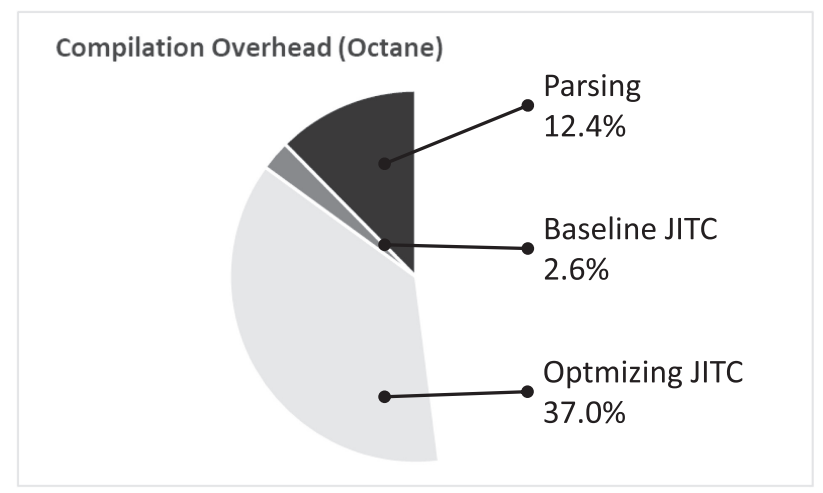

Fig. 1. Compilation overhead of JavaScript engine during the whole JavaScript execution time, for Octane benchmark.

overheads of parsing, baseline JITC, and optimizing JITC. Moreover, reusing the optimized code early, even from the first invocation, can lead to additional performance benefits. Although the idea is straightforward, there are a few challenging issues for reusing the optimized code. For example, we need to relocate many dynamic addresses in the optimized code such as the hidden class addresses, which is not as simple as relocating static addresses in the baseline code. We also need to validate the speculations used during the profile-based optimization in the previous run, to confirm we can reuse the code in the current run. One issue is that sometimes it is impossible to reuse the code since an object specified in the check code for the speculation cannot be identified in the current run (maybe it is not even yet created when we are about to execute the code). Finally, the optimizing JITC can re-compile a function more than once during execution, so which version to save and reuse for AOTC is also an issue. We resolved these issues and implemented the proposed AOTC on the WebKit JSC engine. Our AOTC shows a much higher performance than the original JSC. As far as we know, our work is the first AOTC that reuses the profile-based optimized code generated by the aggressive optimizing JITC of modern JavaScript engines.

The rest of this article is organized as follows. Section 2 contains the background information on modern JavaScript engines and the optimizing JITC. In Section 3 we describe our AOTC approach and shows its design and implementation. In Section 4 we present the experimental results, and in Section 5 we review related work. We conclude in Section 6.

\section{BACKGROUND}

\subsection{JavaScript Engine Architecture}

Modern JavaScript engines generally adopt multi-tier architecture, which is composed of multiple execution tiers including the interpreter and the JITCs. These tiers operate on a functionby-function basis. Figure 2 represents a typical execution process of modern JavaScript engines, such as WebKit's JSC [3], Chrome's V8 [10] and Firefox's SpiderMonkey [26]. Since JavaScript is distributed as the source code format, the JavaScript source code should first be translated before being executed. In the first tier, the parser translates the source code for an invoked JavaScript function to the bytecode. Then, the interpreter initially executes the bytecode to ensure quick start of JavaScript execution. When the function is repeatedly called and considered warm (10 times in JSC), the baseline JITC is triggered to compile the bytecode into simple machine code with minimal optimization (second tier). The baseline JITC also inserts the instrumentation code to collect some profile information. If the baseline code is executed enough to collect stable profiles (66 times in 


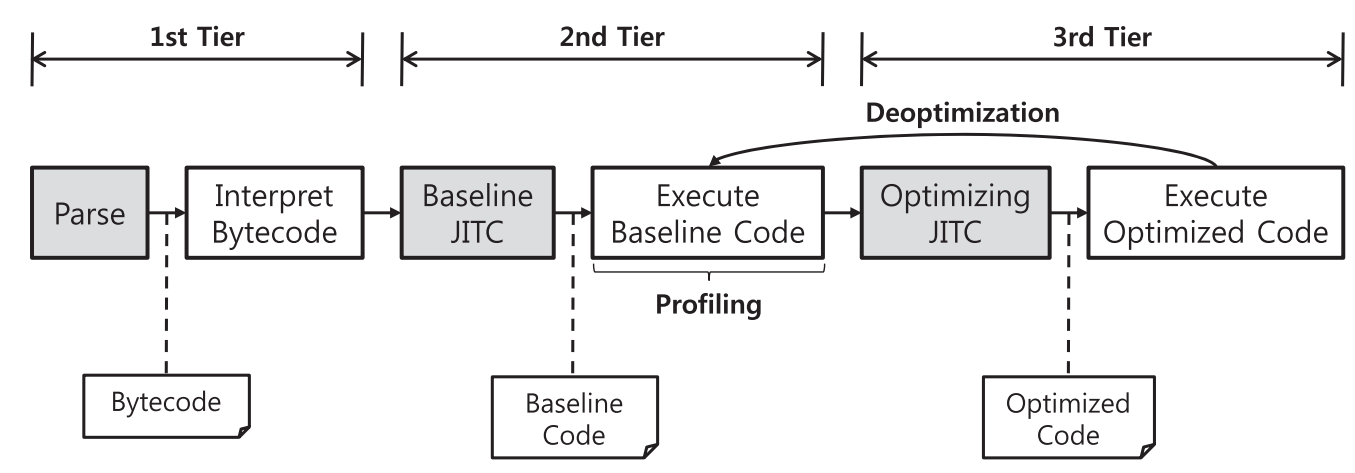

Fig. 2. Multi-tier architecture of JavaScript engines.

JSC), the function is considered hot, initiating the third tier. Now, the optimizing JITC recompiles the bytecode using many optimizations, and the most powerful one is profile-based optimization, which generates speculative code. If some speculation fails, however, de-optimization occurs to go back to the baseline code. The third tier will be explained in Section 2.2. More optimization tiers can be added after the third tier to optimize even hotter JavaScript functions (e.g., FTL in JSC).

\subsection{Profile-Based Optimizations}

Optimizing JITC performs various optimizations such as common sub-expression elimination, loop invariant code motion, function in-lining, etc., often employed in the static compilers. One thing to note is that the optimizing JITC also aggressively performs profile-based optimizations.

JavaScript is a dynamic-typed, object-oriented language. The type of a variable is changeable during execution. The properties (fields) of an object can also be dynamically inserted or deleted. The profile information the baseline code collects is mostly related to dynamic behaviors such as the type of variables or the shape of objects during execution. Then, the optimizing JITC generates code specialized for these profiles, speculating that the same behavior will be repeated in the future.

Figure 3 shows an example JavaScript source code along with the baseline code and the optimized code. The source code shows that a global variable glob is first initialized to an integer 1 . Then, the function $f o o()$ is frequently called in a loop, thus being optimized eventually by the optimizing JITC. $f o o()$ is repeatedly called with an argument object whose $x$ property is initialized to an integer 2 (i.e., foo(\{x:2\})). What $f o o()$ does is add two operands, the argument object's $x$ property and glob. The baseline code of $f o o()$ generates each case of addition code for each operand type to handle dynamic types, as shown in Figure 3.

From the profile information gathered during the baseline code execution, the optimizing JITC recognizes the following. First, glob is initialized once and remains unchanged. Second, the argument object passed to $f o o()$ always has the identical shape where the only property $x$ is located at the first place (offset 0). Finally, two operands of the add operation, glob and $x$ property, are integer values.

Based on this information, the function $f o o()$ is optimized as follows. First, loading of each operand of the add operation is optimized. For the $x$ property of the argument, we need to generate code that loads the value of property $x$. In general, JavaScript engines make use of hidden class, a list of properties and their offsets in the object, to represent the shape of an object. So, every object has a pointer to its hidden class, and to access a property, we should first find the location of the property from the hidden class. However, we observed that $f o o()$ is always called with the identical-shaped object, so the argument object always has one kind of hidden class. Therefore, the optimizing JITC generates the code that directly loads the property $x$ at the fixed location 


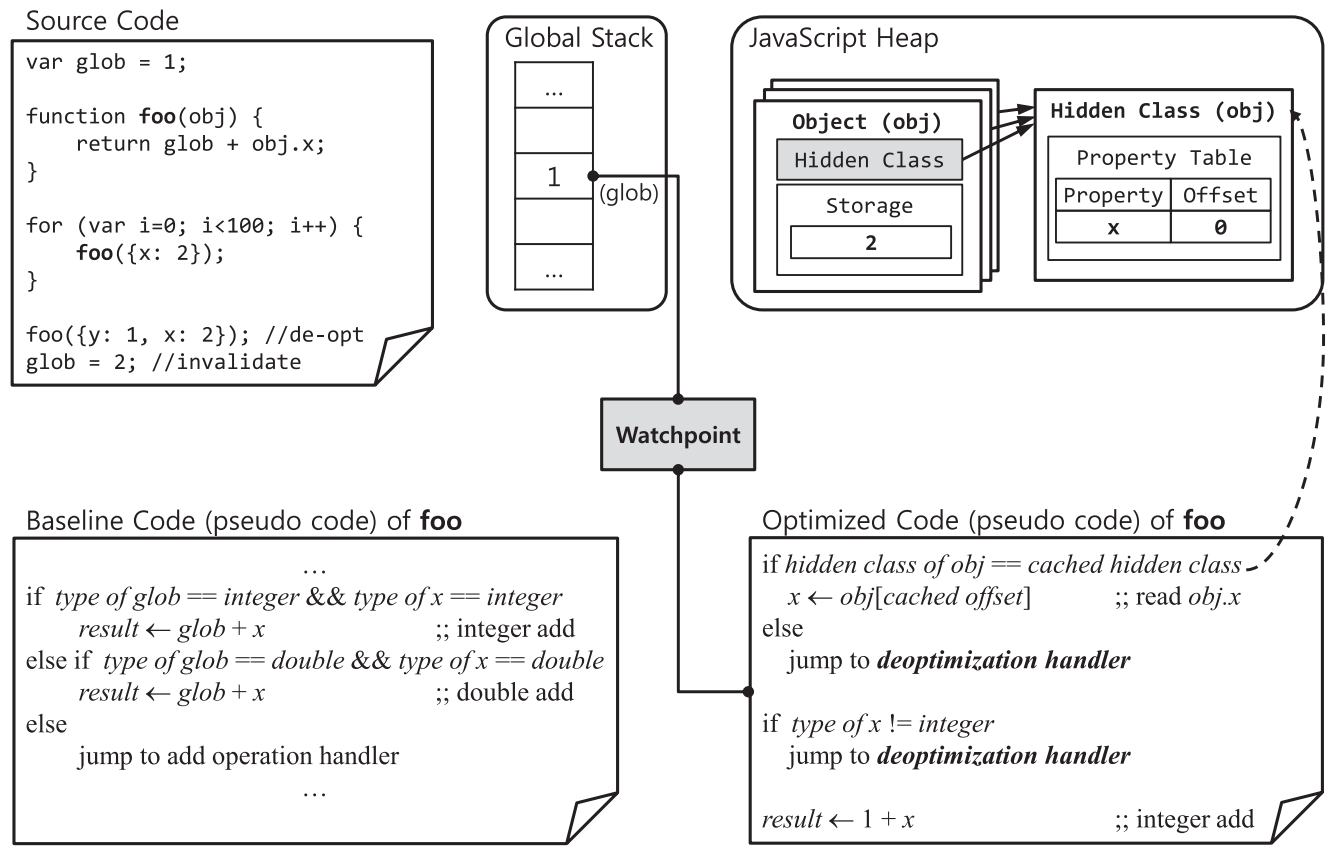

Fig. 3. An example of JavaScript source code with the baseline code and the optimized code for the function foo().

(cached offset 0 ) in the object without looking up the hidden class, expecting the argument will be the identical-shaped object in the future. This optimization of property access is known as inline caching [18]. Of course, this speculation may be invalid in the future, e.g., function foo() may be called with another-shaped object. To validate each speculation, the optimizing JITC also inserts guard code. In this case, the address of the observed hidden class is cached and compared to that of the hidden class of the current argument object. If two addresses are different, it means anothershaped object is passed as an argument (e.g., by $f o o(\{y: 1, x: 2\})$ in the source code of Figure. 3). It triggers a recovery mechanism called de-optimization, which stops the execution at that guard point in the optimized code and resumes the execution at an equivalent point in the baseline code with no speculation, thus continuing the execution. This is actually handled by the de-optimization handler invoked in Figure 3, which switches the execution to the baseline code by recovering the baseline code stack. One thing to note is the optimized code of a function is not discarded right away, since the speculation might be valid next time when $f o o()$ is called. When there are many de-optimizations due to the failure of the guard code, the optimized code is finally discarded, and thereafter the baseline code is used instead. However, if the baseline code is executed frequently again, the function will be recompiled by the optimizing JITC, now with additional profiles gathered during the baseline code execution.

For the other operand glob, the optimizing JITC speculates that glob is a constant because glob is initialized once and unchanged. So, the value of glob (integer 1) is directly used in the add operation rather than generating the code of accessing and loading the value from the global stack. To guard the speculation on the constant value of glob, the JavaScript engine sets up a watchpoint for glob, which obviates the guard code; when there is an update for the variable, the JavaScript engine detects it immediately and discards any optimized code based on the speculation for the constant value. In Figure 3, the watchpoint monitors the change of the glob variable and has a list of 

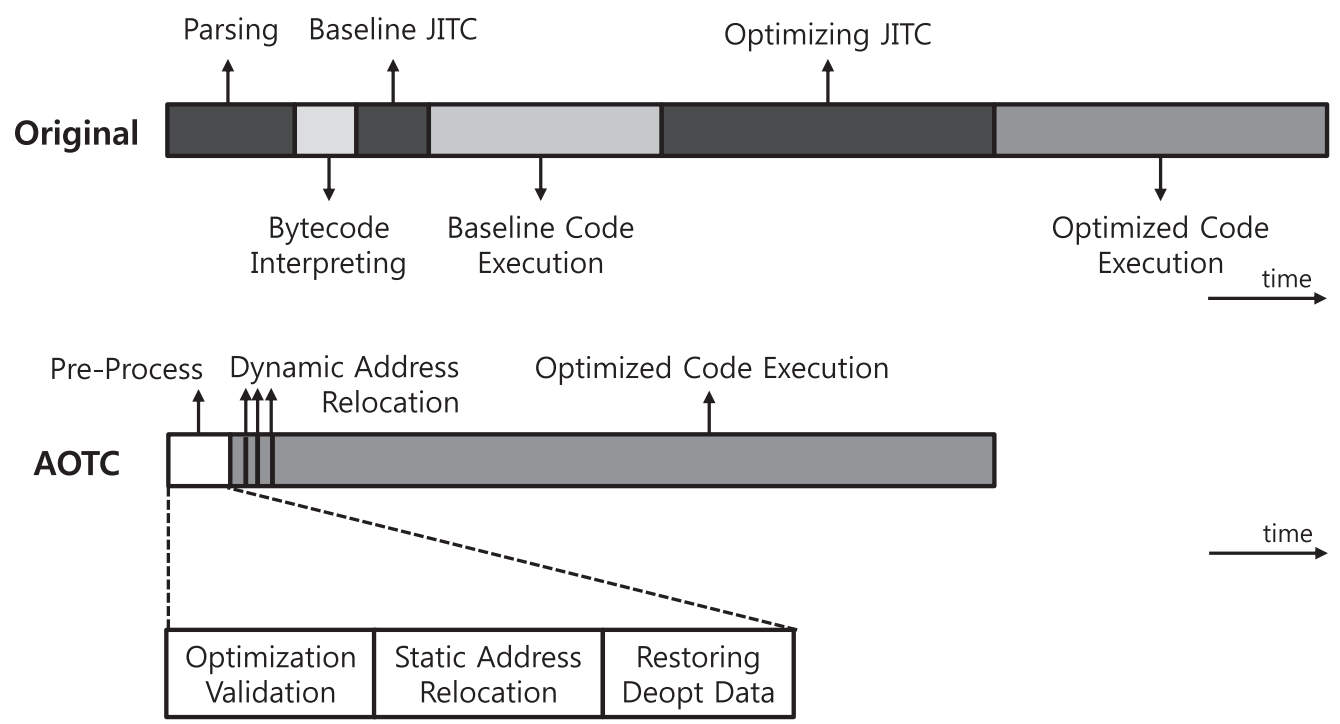

Fig. 4. Process of reusing the optimized code.

functions optimized with the monitored value. If glob is modified (as in the last line of the source code in Figure 3), the watchpoint fires. Then, the optimized code of each function in the list is discarded and the baseline code is used instead, since the optimized code is no longer valid. After the invalidation, every invocation of function $f o o()$ is executed in the baseline code; even when $f o o()$ is recompiled, the watchpoint is not used again since it is invalid. A watchpoint is allocated only for those values that can be accessed globally, such as global variables or prototypes because it is inefficient to monitor every value.

Finally, for the addition operation, the optimizing JITC generates code specialized for integer adds, based on the profile information. Before the addition code, there is guard code that checks the type of the property $x$ and jumps to the de-optimization handler if it is not an integer. Type check for glob is unnecessary because glob is already speculated as 1 and monitored by the watchpoint.

\section{AOTC FOR REUSING OPTIMIZED CODE}

In the previous section, we showed that the optimizing JITC performs many profile-based speculations. In this section, we describe the proposed AOTC, which reuses the speculated code. For the rest of the article, previous run means the run where we save the code for AOTC, while current run means the run where we reuse the saved code.

\subsection{AOTC Framework}

Our proposed AOTC framework works as follows, for each use of a function in the previous run:

(1) For a function compiled by the optimizing JITC, we save its optimized code in the AOTC file for reuse in the subsequent runs. We expect that reusing the optimized code can give a higher performance benefit in two ways:

- We can skip the whole compilation process, thus removing the compilation overhead completely.

- We can execute the optimized code from the first invocation of a function, rather than going thru the all compilation tiers (see Figure 4 and compare). 
(2) For a function compiled by the baseline JITC, we do not save the baseline code in the AOTC file due to three reasons. First, the compilation overhead of the baseline JITC is trivial as seen in Figure 1, so skipping it has little impact. Second, the performance impact of executing the baseline code instead of interpreting the bytecode would be negligible since the baseline code is not optimized at all, while interpretation is done only for first 10 times of function execution, so replacing the interpretation by the baseline code execution would make a little difference. Finally, the baseline code is much larger than the optimized code (our evaluation shows that the baseline code is 3.1 times larger), causing a high space overhead for AOTC.

(3) For a function executed in the previous run, but not compiled by the optimizing JITC (including the function compiled by the baseline JITC in (2) above), we save its bytecode in the AOTC file. This will remove the parsing overhead in the next run, which is substantial as seen in Figure 1.

Consequently, we save the bytecode and the optimized code in the AOTC file, considering both the performance impact and the space overhead. When a function is called for the first time, the AOTC framework checks to determine if the compiled code of that function exists in the file. If there is none, the JavaScript engine parses the function and interprets the bytecode as usual. If the bytecode or optimized code of the function exists, our framework loads and executes it directly. When we save the optimized code in the AOTC file, we also save its bytecode because the optimized code needs to access some JavaScript objects included in the bytecode during execution, such as the number or the string objects. Also, the bytecode is needed to generate the baseline code in case of de-optimization during the execution of the optimized AOTC code, because our AOTC does not reuse the baseline code.

\subsection{How to Reuse the Optimized Code}

The optimized code in the previous run is not directly executable in the current run in its present form, since the execution context and timing is completely different. This requires some modification of the code before execution in the current run, or slightly different code needs to be generated by the optimizing JITC. Some of these issues are challenging and complex, and will be addressed in from Section 3.2.1 to 3.2.5.

3.2.1 Reusing De-optimization Data. When a function for which we save the optimized code is called for the first time, we need to perform three pre-processing steps before executing the optimized code, as depicted in Figure 4. The simplest one is restoring the de-optimization data, which is needed when de-optimization occurs during the execution of the optimized code. That is, since register-allocated values in the optimized code should be re-allocated to the baseline code stack in case of de-optimization, we save the mapping information of register-allocated values in the AOTC file and restore them.

3.2.2 Watchpoint Validation. Watchpoint-based code speculated with a constant value discussed in Section 2.2 cannot be reused directly. That is, since there is no explicit guard check in the optimized code, we first need to validate if the code can really be used in the current run, and then set up the watchpoint appropriately.

For example, in Figure 3, glob is speculated as a constant value 1 in the optimized code of function $f o o()$. When $f o o()$ is called for the first time in the current run, we first need to check if glob is indeed 1, before executing the optimized code. glob might have a different value if the current run takes a different execution path from the previous run, or if it is assigned by the result of a random function call even when the same path is taken. Maybe, glob is undefined initially and 


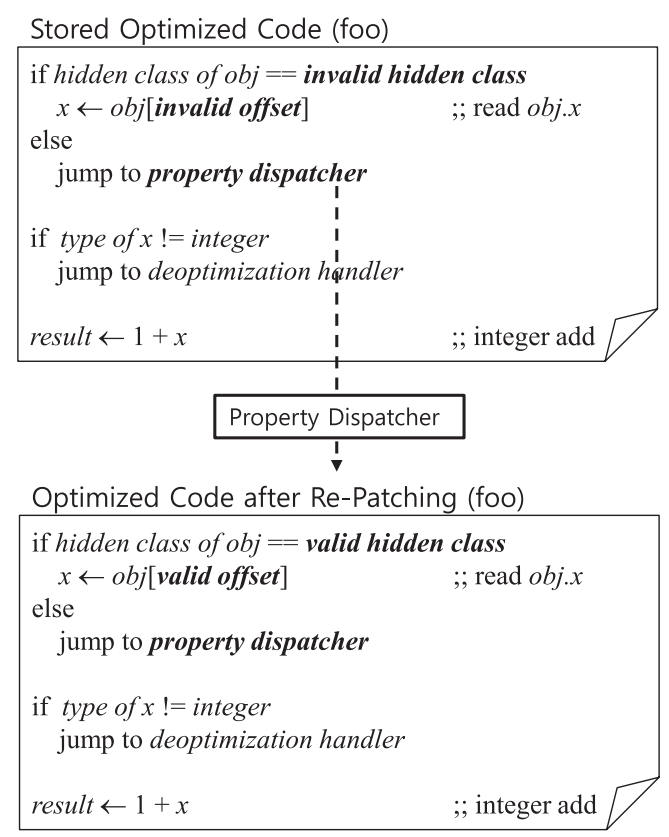

Fig. 5. Process of dynamic address relocation, which dynamically re-patches the optimized code.

later defined just before we save the optimized code (in this case the validation will fail when the function is called for the first time). To validate the watchpoint-guarded speculation, we save the previous value in the AOTC file and compare it to the current value. If validation succeeds, we link the optimized code to the corresponding watchpoint to monitor the value, and allow execution of the optimized code. If not, we discard the optimized code and reuse the bytecode. Watchpoint validation is done as a pre-processing step as shown in Figure 4.

3.2.3 Address Relocation. The optimized code embeds many address values of objects. Since these addresses are not fixed across each run, they should be relocated to the valid address values before the optimized code is reused. For example, the optimized code in Figure 3 includes two address values: one for the de-optimization handler and the other for the hidden class. The de-optimization handler is a static routine, pre-defined in the JavaScript engine. To handle the relocation of these static addresses, we save the location and type of embedded addresses in the AOTC file, and the AOTC framework relocates each embedded static address to the valid address of the current run. Static address relocation is handled during the pre-processing steps before executing the stored optimized code, as shown in Figure. 4.

Unfortunately, we cannot relocate the address of the hidden class in this way. Hidden class objects are dynamically allocated in the heap during runtime. When $f o o()$ is called for the first time in the current run, we cannot easily locate the correct hidden class object in the heap, or the hidden class object might not have been created yet (if obj were created inside foo(), its hidden class object is not yet available just when $f o o()$ is called). To handle this dynamic address relocation, we modify the optimizing JITC to generate the code that can dynamically re-patch itself as shown in Figure 5. That is, the check code includes an invalid hidden class address intentionally, so it fails initially and jumps to the property dispatcher routine. The property dispatcher is also a predefined routine that will re-patch the invalid hidden class address and the offset of the property $x$ by the valid values, by accessing the hidden class from the current obj (using the hidden class 


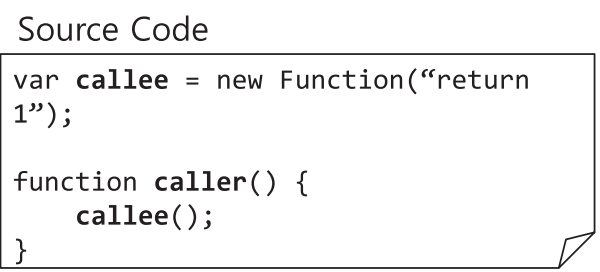

Optimized Code (caller)

if callee object $==$ cached callee object
body of callee $;$ function inlining
else
jump to deoptimization handler

Fig. 6. An example of function in-lining.

pointer inside $o b j$ ). In this way, the next execution of the optimized code can work correctly and efficiently if an identical-shaped object is encountered. When a different-shaped object is met, we jump to the property dispatcher again to re-patch the invalid hidden class address. If the re-patch request is frequent (two in our current implementation), it means that the shape of the object is unstable. In this case, we patch the code to jump to the de-optimization handler to give up using the optimized code.

There are other dynamic addresses in the optimized code for the engine internal objects such as the code block, but we can relocate them more easily. Dynamic address relocation is done mainly during the first execution of the optimized code of the function, as depicted in Figure 4.

3.2.4 Disabled Optimizations. Despite dynamic re-patching, our AOTC cannot easily validate two profile-based optimizations. One is function in-lining, which embeds the body of a callee function inside the caller function to reduce the function call overhead. In JavaScript, a function is an object. So, in the optimized code, the address of an in-lined callee object is cached to check if the current callee function is identical to the in-lined function, as shown in the example of Figure 6. To relocate the address of callee() for AOTC, we would first have to validate if the in-lined body of the callee() is really that of the callee() function invoked in the current run. The function name "callee()" is not enough for validation since a function object can be reassigned during execution and even anonymous functions exist in JavaScript. We can try to compare the body of both callee functions for validation, but there can be more than one function that has the same body during execution. Dynamic re-patching might be used by compiling the body of the current callee thru the optimized JITC and replacing the in-lined code by the current callee's code, yet it is too complicated since compiling the callee only and merging it with the optimized code of the caller is complex (in fact, it might not even fit to the space of the previous, in-lined callee).

The other optimization is watchpoint-based speculation for constant objects (instead of primitive constants described in Section 3.2.2). This optimization is mainly used for the constant prototype objects in JSC (e.g., Math.random()). To verify the optimization, we should save the speculated object and compare it with the current object. This is too burdensome since we must save all properties of the object and compare one-by-one with the current object's properties.

Both optimizations have a similar issue as the relocation of the hidden class address in Section 3.2.3, since we first need to identify the object in the current run corresponding to the object for which speculation was done in the previous run. The difference is that hidden class can be easily traced starting from the object (via the hidden class pointer), while in-lined objects or constant objects have no starting point from which to trace. Therefore, in the current implementation we decided to disable these optimizations in the JITC when we generate the optimized code for AOTC. We found that their disabling affects the performance only by $4 \%$, which seems to be small.

3.2.5 Optimized Code Version to Reuse. A JavaScript function can be compiled several times by the optimizing JITC. For example, if the execution of the optimized code incurs de-optimization frequently (100 times in JSC), The JavaScript engine regards the code as no longer valid and discards it. 


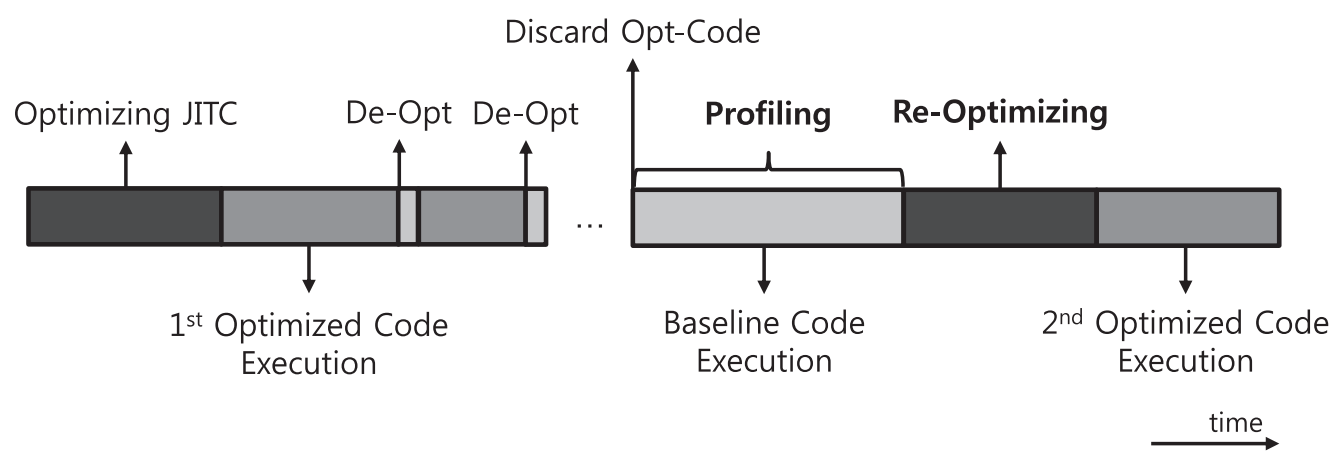

Fig. 7. Process of re-optimization.

Thereafter, the baseline code is executed, collecting more profile information. If the baseline code is executed enough again (66 times in JSC), the optimizing JITC re-compiles it based on newly accumulated profile information as shown in Figure 7. This raises an issue of which optimized version we save for AOTC.

The last optimized code would cause fewer de-optimizations in the current run than the first optimized code since it is based on more profile information, thus having more check code and specialized code that can deal with more runtime cases (e.g., specialized for integer-add vs. specialized for double-add as well as integer-add). On the other hand, execution of more check code might have a slightly negative affect on the performance. We evaluated both the first optimized code and the last optimized code in our experiments.

One tempting idea is changing the tier-transition thresholds to obtain a better quality code to save for AOTC. For example, we can reduce the de-optimization threshold to discard the optimized code earlier, and reduce the hotspot detection threshold to boost the re-compilation by the optimizing JITC earlier with more profile information. Unfortunately, these attempts were not successful and we believe that the default threshold values in JSC are highly engineered ones, thus not easily adjustable for AOTC.

\subsection{Implementation of AOTC on JavaScriptCore engine}

We implemented our AOTC on the JavaScriptCore (JSC) of WebKitGTK version 2.11.1 (released on 2015-11-03), a commercial web engine used as a code base of Apple's Safari browser. We discuss some implementation issues here.

3.3.1 JSC Optimizing Just-in-Time Compiler (JITC) for AOTC. There are two optimizing JITCs in JSC: DFG (data flow graph) and FTL (fourth-tier low-level virtual machine (LLVM)). DFG is enabled first when the baseline code becomes hot, and FTL is enabled when the DFG code is even hotter. For our AOTC, we use the DFG as the optimizing JITC. FTL translates the bytecode to the LLVM intermediate representation (IR) first, then generates the optimized code using the LLVM backend. Since AOTC requires relocation of addresses in the optimized code as well as other updates, AOTC for FTL would require changing the LLVM backend, which is beyond the scope of JavaScript optimization. Actually, FTL is rarely enabled, especially for web apps during app loading time.

3.3.2 Address Relocation of String Objects. For character strings in the JavaScript source code (e.g., "glob", "obj", " $x$ " in $f o o()$ of Figure 8), JSC internally represents each string by wrapping it into a StringImpl object in the browser heap (this is different from JavaScript string objects in the JavaScript heap). There is a string table in JSC which has the address of a newly allocated StringImpl object, as depicted in Figure 8. The table is used for JSC to allocate a single StringImpl 

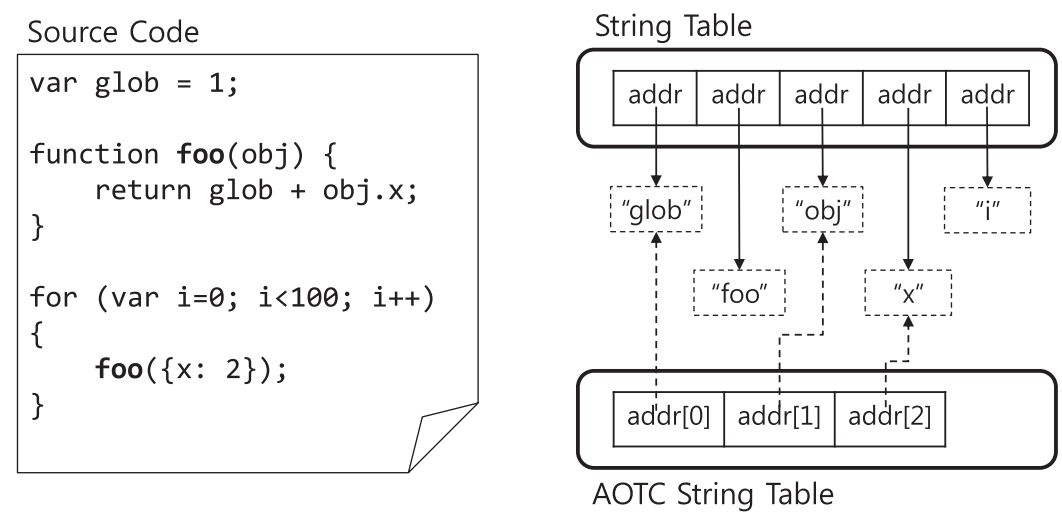

Fig. 8. An example of string table and AOTC string table.

object for each unique string value, to avoid duplication of the same string values in memory (i.e., if the same strings are used in a function, their corresponding StringImpl objects will be identical).

The optimized code may embed the address of a StringImpl object to use the string value. By comparing the address of StringImpl, the optimized code can easily check if two string values are identical. For AOTC, we need to relocate these StringImpl addresses, so we made another table called the AOTC String Table as in Figure 8. The AOTC string table contains only those addresses of StringImpl objects embedded in the optimized code of a function without any duplicated addresses. We save the AOTC table with the actual character string of each entry. When saving the optimized code, we mark the index of the AOTC string table for each embedded address. When reusing the optimized code, we first restore the AOTC string table by allocating a StringImpl object for each entry in the table (which will be added to JSC's string table too). Then, we relocate the embedded address based on the marked index number. By using the AOTC string table, we can save only necessary, non-duplicated character strings. One issue is that we save normal 8-bit ASCII character strings, but some programs exceptionally use 16-bit ASCII character strings obtained by computation of ACSII strings. These extended ASCII strings are complicated to save and restore, so we do not apply AOTC for a function that uses such strings.

3.3.3 Data Compression. For a resource-restricted mobile environment, space usage would be a critical issue, so AOTC should use a minimal storage for saving the AOTC file. To reduce the size of the AOTC file, we used a compression technique called LZ4 [6], a lossless compression algorithm focused on fast compression. For compression, we allocate additional data buffer to compress and decompress the AOTC data as a whole. When saving the code, every AOTC data is accumulated in the buffer during the execution. At the end of execution, the buffer is compressed once by the compression algorithm, and then finally stored into the AOTC file. When reusing the stored code, the entire stored data is loaded into the buffer from the file and decompressed. Then, the data buffer is accessed during the execution when the necessary code or data is needed. Data compression makes AOTC approach more feasible for a space-limited environment, but decompression overhead is added to the execution time. We evaluate and discuss the decompression overhead in Section 4.

3.3.4 Source Code Hashing. If the JavaScript source code for which we made an AOTC file is modified, the AOTC framework should detect this to avoid reusing the wrong code. To solve this issue, we also cache the hash value of each JavaScript code together with the compiled code. Fast hash algorithm [17] is employed to minimize the calculation overhead. When reusing the compiled 
Table 1. List of the Web Apps Experimented

\begin{tabular}{|l|l|l|}
\hline Web App & Description & Framework \\
\hline Hello-Backbone & $\begin{array}{l}\text { Simple tutorial of “hello world" example } \\
\text { (http://arturadib.com/hello-backbonejs/1.html) }\end{array}$ & $\begin{array}{l}\text { Backbone.js, jQuery, } \\
\text { Underscore.js }\end{array}$ \\
\hline Memory-Game & $\begin{array}{l}\text { Memory game of finding a matching pair of cards } \\
\text { (http://igorminar.github.io/Memory-Game/app/ } \\
\text { index.html) }\end{array}$ & AngularJS \\
\hline Painter & $\begin{array}{l}\text { Web painter } \\
\text { (http://lislis.sakura.ne.jp/canvas/paint/paint.html) }\end{array}$ & jQuery \\
\hline Pathfinding & $\begin{array}{l}\text { Find the shortest path between two points } \\
\text { (http://qiao.github.io/PathFinding.js/visual/) }\end{array}$ & jQuery \\
\hline StarterKit & $\begin{array}{l}\text { Starter kit for EmberJS framework } \\
\text { (https://github.com/emberjs/starter-kit) }\end{array}$ & Ember.js, jQuery \\
\hline Todo-Backbone & $\begin{array}{l}\text { Todo list app with BackboneJS framework } \\
\text { (http://todomvc.com/examples/backbone/) }\end{array}$ & $\begin{array}{l}\text { Backbone.js, jQuery, } \\
\text { Underscore.js }\end{array}$ \\
\hline
\end{tabular}

code, the AOTC framework first calculates the hash value of the current source code and checks it with the cached value. If the two values are inconsistent, this means that the source code is changed. In this case, we discard the stored code and run the modified source code by the multitier architecture as usual. After the execution, we save the newly compiled code to reuse it for AOTC.

\section{EVALUATION}

\subsection{Experimental Setup}

We evaluated our AOTC on a quad-core $3.4 \mathrm{GHz}$ Intel i7 processor with 16GB RAM, running Ubuntu 15.10. We experimented with the industry-standard Octane JavaScript benchmark and six web apps. The Octane JavaScript benchmark consists of 17 tests that cover various computational intensive and real-world-based workloads, such as the PDF reader(pdfjs), 2D and 3D physics engines (Box2DWeb and Mandreel), and the full compiler, which compiles the superset of JavaScript to the pure JavaScript (Typescript). The six web apps we chose to experiment with are the apps from various domains, all of which are programmed with one or more popular JavaScript frameworks as listed in Table 1.

We evaluated three AOTC configurations:

- AOTC_Bytecode (AB): reuse only the bytecode

- AOTC_All_First (AAF): reuse the bytecode and the first optimized code

- AOTC_All_Last (AAL): reuse the bytecode and the last optimized code

All presented results are the average of 10 measurements.

\subsection{Performance Analysis}

We first mainly discuss the evaluation against the Octane benchmark. Figure 9 shows the performance impact of each AOTC configuration, compared to the performance of the original JSC engine. The average speedup is $1.15 \mathrm{x}(\mathrm{AB}), 1.81 \mathrm{x}(\mathrm{AAF})$, and 1.99x (AAL), respectively. All three AOTCs improve the performance for all programs (except for slight degradation in AAF for Richards).

The performance improvement of AOTCs comes primarily from the reduction of the compilation overhead. To analyze this impact, we break down the JavaScript execution time into the 


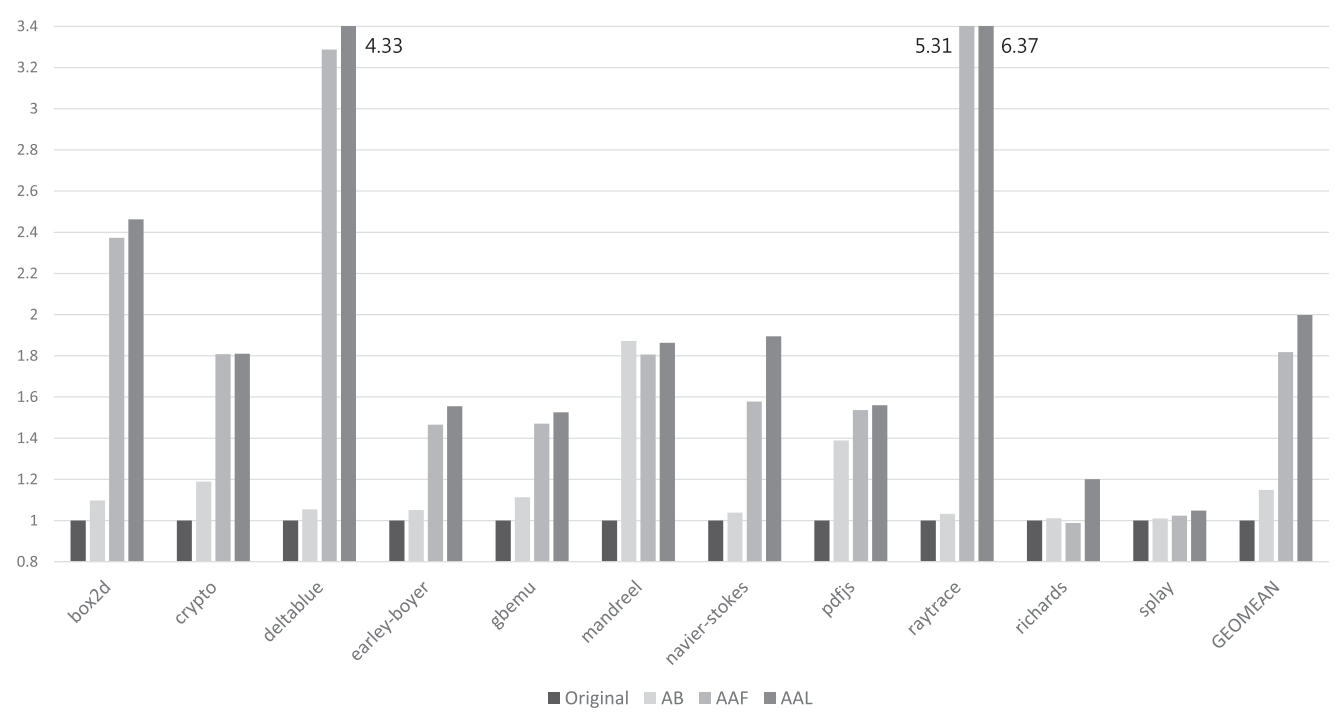

Fig. 9. Speedup of AOTC for Octane benchmark.

parsing time, the baseline JITC time, the optimizing JITC time, the preprocessing time for AOTC, and others, as depicted in Figure 10. AAF or AAL reduce the compilation overhead much better than $\mathrm{AB}$ since they remove much of the optimizing JITC overhead. They cannot eliminate the total compilation overhead though. One reason is that recompilations still occur when we reuse the optimized code due to de-optimization. The other reason is that we could not apply AOTC to some functions, as we mentioned in Section 3, so they are compiled by the JITCs.

Early execution of the optimized code starting from the first function invocation can also contribute to the performance improvement. We estimated this impact by subtracting the reduced compilation overhead from the total reduced execution time. Some programs show a tangible impact such as box2d (19.9\%), DeltaBlue (106.2\%) and Navier-Stokes (41.5\%). Other programs have little impact, so their performance improvement is mostly due to the reduced compilation overhead.

Table 2 compares parsing, baseline compilation, and optimizing compilation counts for the original run and the AAL run. We can see that AAL has significantly fewer compilation counts compared to the original run, as expected. Table 2 also shows the number of optimized functions saved in AAL. For some benchmarks, this number is higher than the number of optimizing compilations in the original run. We found this is due to the disabled in-lining in our version of optimizing JITC used for AOTC. That is, if a caller becomes hot earlier than a callee, the optimizing JITC will compile the caller with the callee being in-lined, then the callee will not be compiled separately even when it becomes hot since it is already in-lined; if in-lining is disabled, both the caller and the callee will be compiled.

Table 2 also shows the number of validation attempts for watchpoints and the number of functions with failed validation. Some programs such as Mandreel or Richards have many watchpoint failures. This makes AAF and AAL performance similar to AB for Mandreel, and makes AAF performance for Richards even worse than $\mathrm{AB}$. Actually, more than half of the stored optimized code could not be reused because of the failed watchpoint validations (AAL for Richards performed better than AAF, though, which seems to be due to fewer de-optimizations; see Table 3). These failures are mostly due to uninitialized or differently initialized global variables when reusing, as discussed in Section 3.2.2. Finally, Table 2 shows that there are quite a few static and dynamic address relocations to reuse the optimized code. 


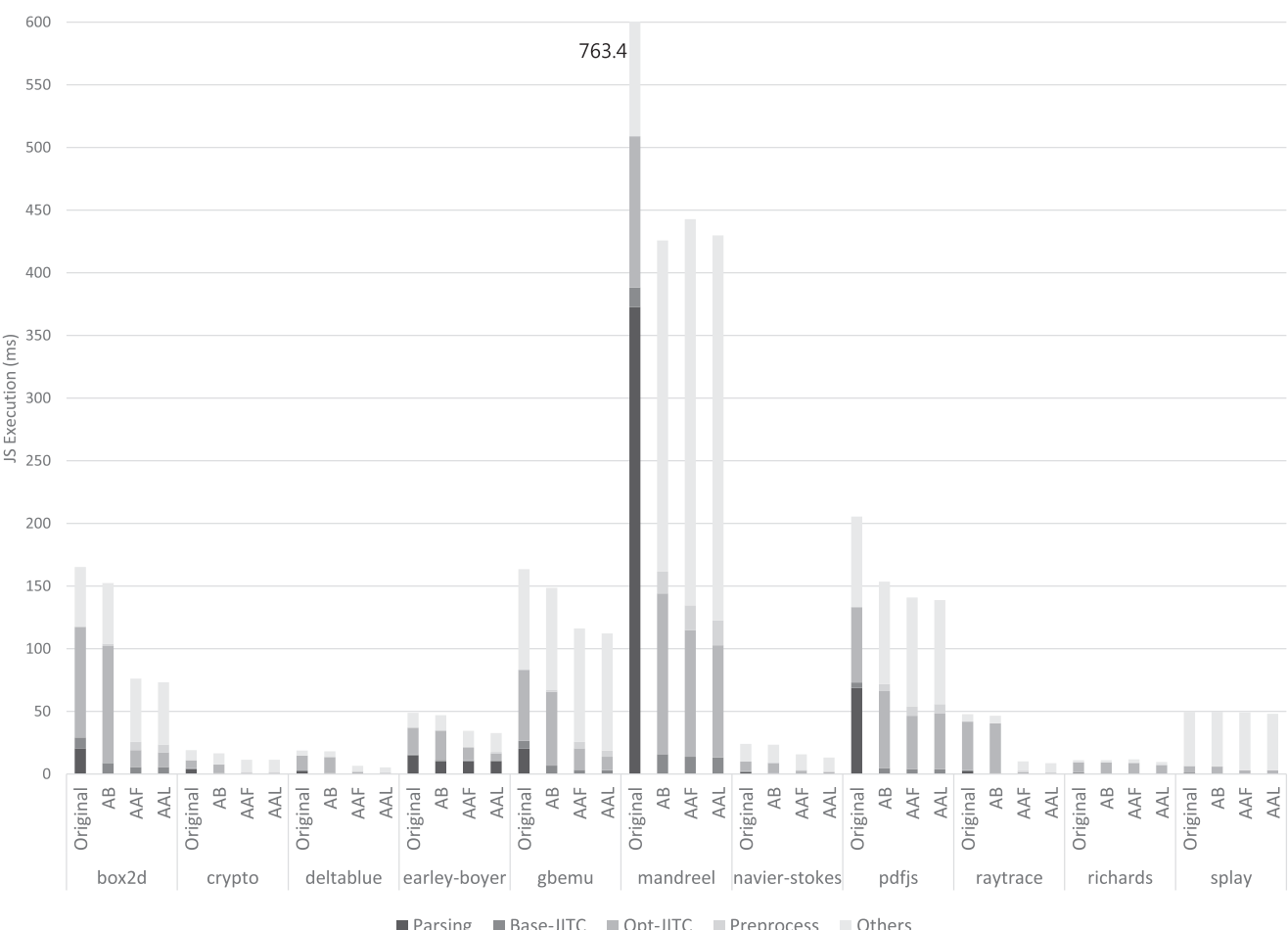

Fig. 10. Breakdown of JavaScript execution for Octane benchmark.

Table 2. Total Number of Each Compilation Process and AOTC-Related Count

\begin{tabular}{|c|c|c|c|c|c|c|c|c|c|c|c|c|}
\hline & & box $2 \mathrm{~d}$ & crypto & deltablue & $\begin{array}{l}\text { earley- } \\
\text { boyer }\end{array}$ & gbemu & mandreel & $\begin{array}{l}\text { navier- } \\
\text { stokes }\end{array}$ & pdfjs & raytrace & richards & splay \\
\hline \multirow{3}{*}{ Original } & Parsing & 332 & 64 & 73 & 88 & 393 & 181 & 29 & 406 & 46 & 34 & 20 \\
\hline & Base-JITC & 213 & 19 & 50 & 36 & 192 & 77 & 10 & 99 & 28 & 17 & 15 \\
\hline & Opt-JITC & 150 & 10 & 42 & 37 & 183 & 68 & 12 & 78 & 25 & 11 & 8.8 \\
\hline \multirow{8}{*}{ AAL } & Parsing & 0 & 0 & 0 & 4 & 1 & 0 & 0 & 9 & 0 & 0 & 0 \\
\hline & Base-JITC & 103 & 11 & 10 & 19 & 49 & 50 & 8 & 62 & 3 & 13 & 8 \\
\hline & Opt-JITC & 9 & 1 & 1 & 11 & 16 & 38 & 2 & 29 & 0 & 14 & 3 \\
\hline & $\begin{array}{l}\text { Functions saved } \\
\text { with Opt-code }\end{array}$ & 134 & 12 & 41 & 31 & 159 & 69 & 10 & 62 & 26 & 17 & 11 \\
\hline & Validations & 187 & 30 & 45 & 95 & 321 & 261 & 27 & 129 & 30 & 38 & 15 \\
\hline & $\begin{array}{l}\text { Functions with } \\
\text { Failed Validation }\end{array}$ & 0 & 1 & 0 & 4 & 0 & 36 & 0 & 4 & 0 & 8 & 1 \\
\hline & $\begin{array}{l}\text { Static Address } \\
\text { Relocation }\end{array}$ & 15956 & 1096 & 1737 & 2040 & 10151 & 9460 & 933 & 5034 & 2699 & 970 & 617 \\
\hline & $\begin{array}{l}\text { Dynamic } \\
\text { Address } \\
\text { Relocation }\end{array}$ & 3210 & 216 & 218 & 218 & 3120 & 3423 & 128 & 864 & 430 & 154 & 80 \\
\hline
\end{tabular}


Table 3. Comparison of De-optimization Count

\begin{tabular}{|c|r|r|r|r|r|r|r|r|r|r|r|}
\hline Deopt-Count & box2d & crypto & deltablue & $\begin{array}{c}\text { earley- } \\
\text { boyer }\end{array}$ & gbemu & mandreel & $\begin{array}{c}\text { navier- } \\
\text { stokes }\end{array}$ & pdfjs & raytrace & richards & splay \\
\hline Original & 7213 & 42.3 & 1174 & 1490 & 2945 & 237 & 11 & 1793 & 204 & 203 & 20.8 \\
\hline AAF & 873 & 35 & 1713 & 2154 & 1236 & 1072 & 11 & 2906 & 302 & 1165 & 42 \\
\hline AAL & 807 & 35 & 5 & 929 & 242 & 206 & 10 & 1827 & 0 & 462 & 42 \\
\hline
\end{tabular}

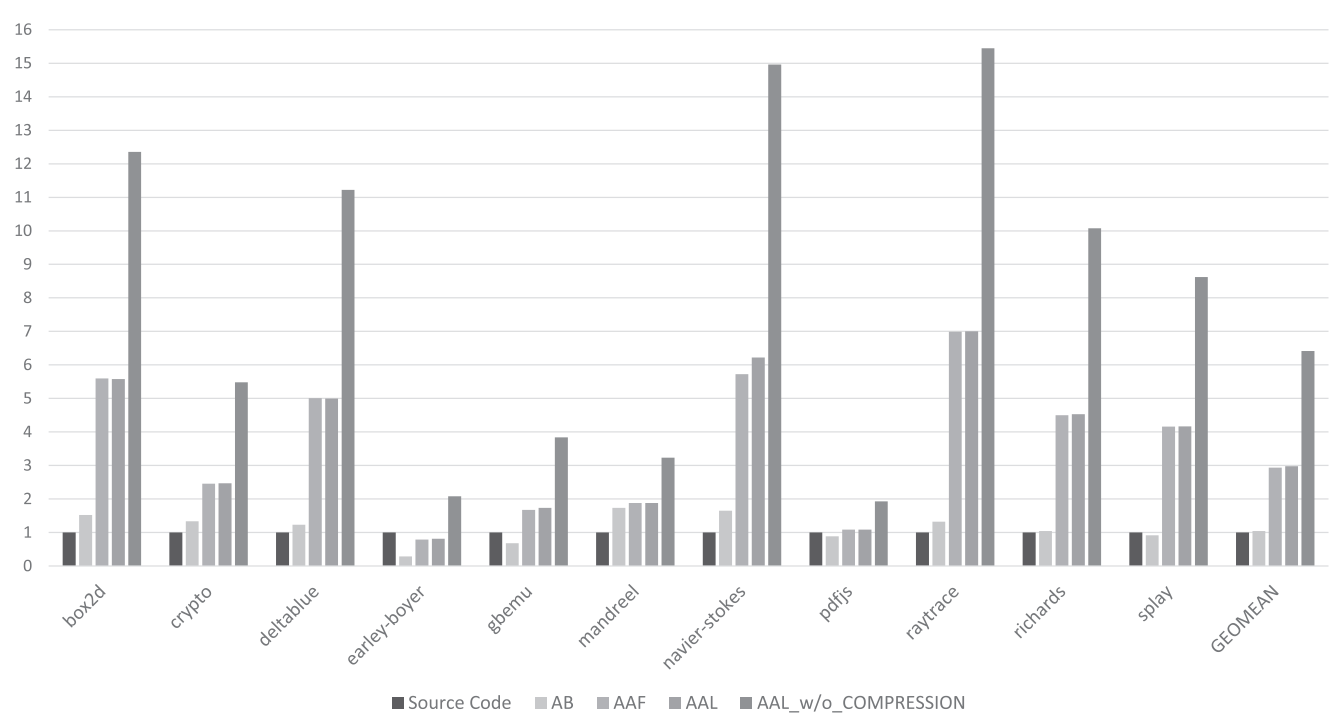

Fig. 11. Storage usage of each AOTC compared to the size of source code.

When comparing AAL and AAF in Figure 9, AAL shows an average performance 1.1 times better than AAF. This is due to fewer de-optimization counts in AAL as shown in Table 3. The highest speedup appeared in DeltaBlue (1.32x), which shows the largest difference in de-optimization counts between AAF and AAL. AAL reuses the last optimized code, more profiled and specialized with more cases, compared to AAF, which reuses the first optimized code with insufficient profiling. Additional type-check overhead in AAL does not seem to affect the performance. In Table 3, we can also see some cases where the de-optimization count is higher in AOTCs, especially in AAF, compared to the original. We found that most of these cases are due to the lack of profiling during the bytecode interpretation. As we explained previously, JSC does not perform profiling during the bytecode interpretation but only during the baseline code execution. This can cause the optimized code fail to deal with those cases occurring during the interpretation, and if so, deoptimization can occur during the initial invocations of such functions. Since AAL is based on more profiles, it might include those missing cases observed during interpretation. Some decrease of de-optimization counts in AOTC is due to the disabled optimizations, which lead to reduced speculations and guard code for them, thus fewer chances of de-optimization.

\subsection{Space and Decompression Overhead}

Figure 11 shows the size of AOTC files compared to the size of JavaScript source code. By using the compression algorithm, all AOTC configurations take a space less than three times of the source code size, on average (which is half of the uncompressed case). The decompression 


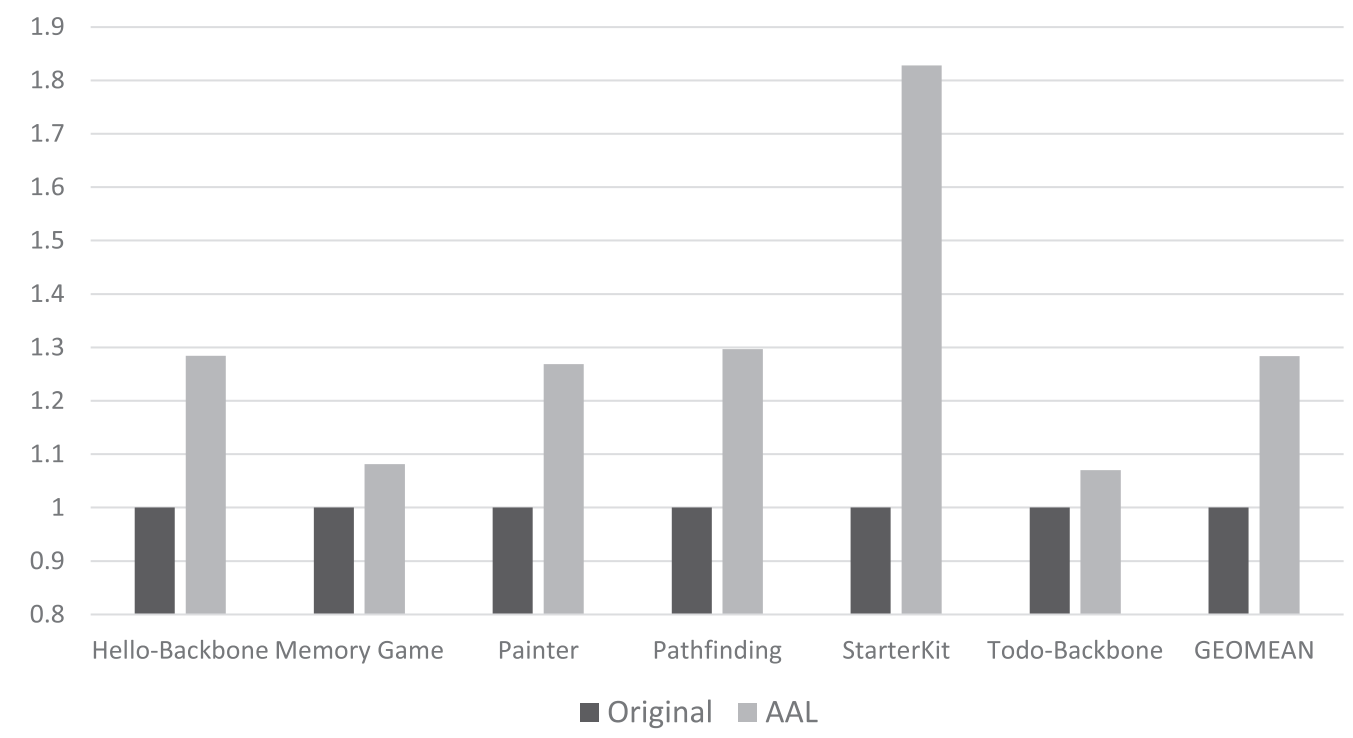

Fig. 12. Speedup of web app loading.

overhead is less than $1 \%$ of the total execution time. Both results show that our AOTC is feasible space-wise.

\subsection{Performance Impact on Web App Loading Time}

We also experimented with six JavaScript-heavy web apps to see if AOTC has an impact on their app loading time, which is the time from the start of app and until the first screen of the app is rendered. Since JavaScript code executed during app loading is mostly for app initialization, including the web framework (e.g., jQuery) initialization, it is usually a repeated task with the same hot functions executed with the same computations, thus suitable for AOTC. Figure 12 shows the performance impact of AAL on app loading time for the six web apps. The speedup is $1.28 \mathrm{x}$ on average, most of which comes from reusing the optimized code during the initialization of the framework (the decompression overhead of AOTC takes less than $0.5 \%$ of the app loading time). Our estimation also shows that three apps show considerable performance improvement by the early execution of the optimized code from the first function invocation, such as Painter (13.5\%), Pathfinding (13.7\%), and StarterKit (16.1\%). Since the app loading performance is more critical to user experience than steady-state performance or benchmark performance, these results indicate AOTC is promising for improving real web experience.

\subsection{Performance Impact on Web Page Loading Time}

Finally, we experimented with four popular web pages selected from the Alexa list [1], to check if AOTC has any impact on their page loading time. Figure 13 shows that the speedup of web page loading by AAL is $7.4 \%$ on average, which is much smaller than that of the web apps, yet still tangible (also, there is no performance degradation). The reason for the smaller impact is that web pages typically have much smaller JavaScript portion than web apps. Furthermore, JavaScript in web pages tends to be executed less repeatedly than benchmarks [36, 38], which means that JavaScript code in web pages are rarely compiled by the optimizing JITC. In fact, we found that most of the performance gain in Figure 13 comes from reusing the bytecode, not optimized machine code. So, it is clear that JavaScript-heavy webs gain more benefit from reusing the optimized code. 


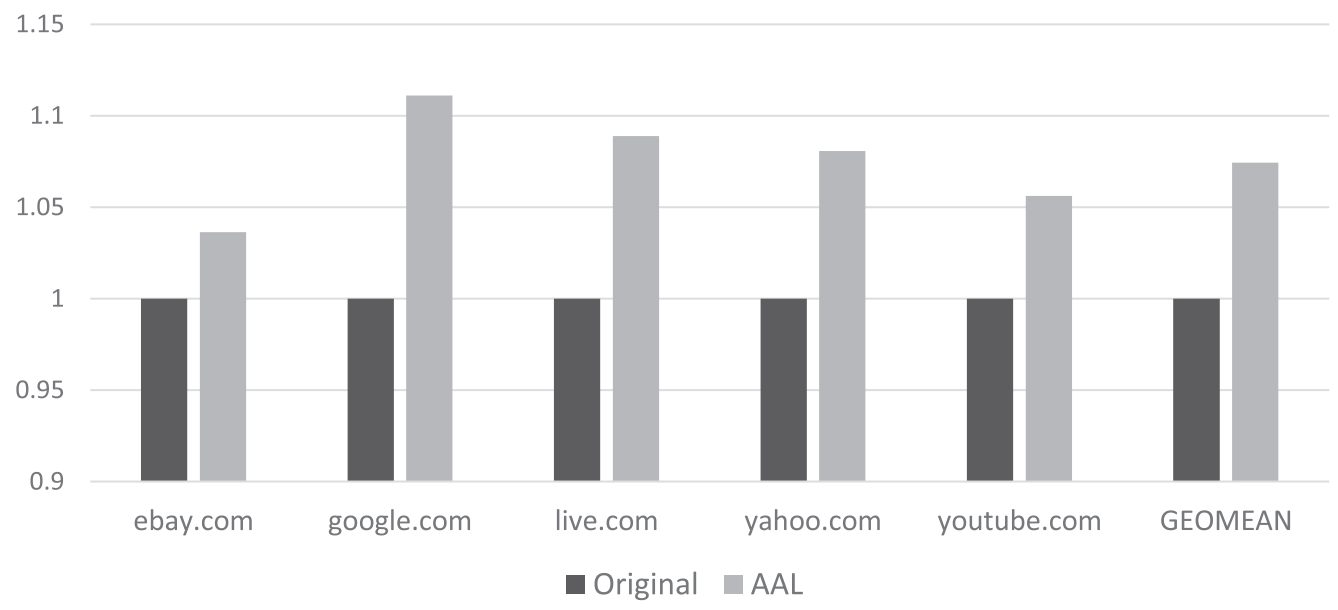

Fig. 13. Speedup of web page loading.

\section{RELATED WORK}

\subsection{Persistent Code Cache}

A persistent code-cache scheme saves the code during one run to reuse it in later executions as a means for amortizing runtime translation costs. Hazelwood and Smith first characterized the optimization persistence where each optimization is repeated during successive program executions in the context of a dynamic optimization system [15]. From experiments on a broader set of applications, this work demonstrates that there is a significant opportunity for leveraging inter-execution persistence (persistence between same program executions). In addition, there are even a few opportunities for inter-application persistence (persistence between different program executions).

Based on the observation of optimization persistence, implementations of persistent code cache have been explored mainly in the domain of binary translation. Reddi et al. describe the persistent code cache for a dynamic binary instrumentation tool, Pin, to extend the scope of code reuse to both inter-execution and inter-application [37]. In particular, each code trace of a program is stored in a file, and library code generated by other programs is also shared between different programs. This method benefits programs with severe startup costs and large code footprint. Bruening and Kiriansky suggest a method of sharing code caches among multiple processes for the DynamoRIO dynamic instrumentation engine [5]. Shared code caches employ the adaptive level of granularity that supports maximum inter-process sharing for unchanging application code while dynamically switching to a fine-grained model that performs for dynamically changing code. This work achieves a significant reduction in memory usage and startup time.

Compared to the previous methods, our approach focuses on reusing the dynamically generated code only for inter-executions since reusing the optimized code is specialized for each program with specific inputs. However, JavaScript frameworks such as jQuery are commonly exploited in real web apps. Therefore, the optimized code of each framework code can be shared between different programs. This approach is left as a promising future task.

\subsection{JavaScript Performance}

There have been many research efforts to improve the JavaScript performance. We review some of them relevant to our work.

asm.js is a strict subset of JavaScript intended to be the target language of translation from statically-typed languages such as C [27]. This language is designed to allow apps written in 
statically typed languages to be run on the web browser while maintaining the performance closer to that of native code. Source-to-source compilers such as Emscripten generates asm.js code with type annotations using subtle syntactic hints [40]. This enables the JavaScript engine to quickly optimize the code without online profiling. However, the syntax of asm.js is very restrictive and is not suitable for hand-written JavaScript code. On the other hand, our approach handles the entire JavaScript language without any restriction.

WebAssembly is a new low-level web standard language, which is similar to asm.js that provides multiple languages with a compilation target so that they can run on the web [14]. Since WebAssembly is a compact binary format with static-type system, it can run faster than JavaScript by initially compiling with existing optimizing JITC. This language is designed to complement and run alongside JavaScript, so it is also an alternative for high performance JavaScript.

Kedlaya et al. describe an approach to reduce the number of de-optimizations by using ahead-oftime profiling (AOTP) [22]. The main idea of this work is to perform offline profiling by running the JavaScript program on the web server. The offline profiler collects information about types and object shapes as well as causes of de-optimizations. Client JavaScript engines then compile hot functions earlier based on the offline profiling information, generating the optimized code to avoid all de-optimizations observed during the offline profiling.

Oh and Moon propose a snapshot-based technique to accelerate web loading sequence (startup of web page until the first screen appears) [30]. They cache snapshots of the JavaScript heap objects generated during the loading time, and restore the heap from the snapshots to skip the execution of load-time JavaScript code. Although this approach represents substantial performance impact, it is suitable only for the loading sequence. In contrast, our AOTC deals with entire JavaScript execution, including the loading time.

\section{CONCLUSION}

This article proposed the ahead-of-time compilation approach to reuse highly optimized code with profile-based optimization for modern JavaScript engines. It can achieve a better performance than existing approaches, which only reuse the bytecode or machine code generated by the baseline JITC. We made the following contributions in this article:

- To the best of our knowledge, we proposed the first approach to reuse the profile-based optimized code for modern JavaScript engines.

- We efficiently resolved the issues related with reusing the optimized code, such as dynamic address relocation and validating previous speculations.

- We implemented our AOTC technique on a commercial JavaScript engine and experimented with the benchmarks, real web apps, and web pages.

- We improved the JavaScript performance by 1.99 times on average, and by 6.36 times on maximum for JavaScript benchmark. We also achieved a tangible reduction of loading time for web apps and web pages.

Future web apps will increasingly include heavy JavaScript frameworks, so their compilation overhead will be critical. We expect our proposed AOTC can make greater performance impact for these apps, as seen from the result of web-loading performance. We also expect AOTC can improve the energy efficiency for the mobile computing environment by sharply reducing the compilation overhead, which is left as a future work.

\section{ACKNOWLEDGMENTS}

We thank Hyeon-Jae Lee for helping our experiment. 


\section{REFERENCES}

[1] Alexa. 2018. The Top 500 Sites on the Web. Retrieved from https://www.alexa.com/topsites.

[2] Apple Inc. 2018. WebKit. Retrieved from https://webkit.org/.

[3] Apple Inc. 2018. WebKit JavaScriptCore. Retrieved from https://github.com/WebKit/webkit/tree/master/Source/ JavaScriptCore.

[4] Igor Böhm, Tobias J. K. Edler von Koch, Stephen C. Kyle, Bjorn Franke, and Nigel Topham. 2011. Generalized justin-time trace compilation using a parallel task farm in a dynamic binary translator. In Proceedings of the 2011 32nd ACM SIGPLAN Conference on Programming Language Design and Implementation (PLDI'11). New York, NY, 74-85. DOI : http://dx.doi.org/10.1145/1993498.1993508

[5] Derek Bruening and Vladimir Kiriansky. 2008. Process-shared and persistent code caches. In Proceedings of the 4th ACM SIGPLAN/SIGOPS International Conference on Virtual Execution Environments (VEE'08). ACM, New York, 61-70. DOI : http://dx.doi.org/10.1145/1346256.1346265

[6] Yann Collet. 2018. LZ4 compression algorithm. Retrieved from http://lz4.github.io/lz4/.

[7] ECMA International. 2015. ECMAScript 2015 Language Specification. Retrieved from http://www.ecmainternational.org/ecma-262/6.0.

[8] Andreas Gal, Brendan Eich, Mike Shaver, David Anderson, David Mandelin, Mohammad R. Haghighat, Blake Kaplan, Graydon Hoare, Boris Zbarsky, Jason Orendorff, Jesse Ruderman, Edwin W. Smith, Rick Reitmaier, Michael Bebenita, Mason Chang, and Michael Franz. 2009. Trace-based just-in-time type specialization for dynamic languages. In Proceedings of the 2009 30th ACM SIGPLAN Conference on Programming Language Design and Implementation (PLDI'09). ACM, New York, 465-478. DOI : http://dx.doi.org/10.1145/1542476.1542528

[9] Google Inc. 2018. Chrome. Retrieved from https://developer.chrome.com.

[10] Google Inc. 2018. V8 JavaScript engine. Retrieved from https://developers.google.com/v8.

[11] Google Inc. 2018. Octane 2.0 JavaScript benchmark. Retrieved from https://chromium.github.io/octane.

[12] L. Guckert, M. O’Connor, S. K. Ravindranath, Z. Zhao, and V. J. Reddi. 2013. A case for persistent caching of compiled JavaScript code in mobile web browsers. In Proceedings of the 2013 Workshop on Architectural and Microarchitectural Support for Binary Translation (AMAS-BT'13).

[13] Jungwoo Ha, Mohammad R. Haghighat, Shengnan Cong, and Kathryn S. McKinley. 2009. A concurrent trace-based just-in-time compiler for single-threaded JavaScript. In Proceedings of the 2009 Workshop on Parallel Execution of Sequential Programs on Multicore Architectures (PESPMA'09).

[14] Andreas Haas, Andreas Rossberg, Derek L. Schuff, Ben L. Titzer, Michael Holman, Dan Gohman, Luke Wagner, Alon Zakai, and J. F. Bastien. 2017. Bringing the web up to speed with WebAssembly. In Proceedings of the 2017 38th ACM SIGPLAN Conference on Programming Language Design and Implementation (PLDI 2017). ACM, New York, NY, 185-200. DOI : http://dx.doi.org/10.1145/3062341.3062363

[15] Kim Hazelwood and Michael D. Smith. 2003. Characterizing inter-execution and inter-application optimization persistence. In Proceedings of the 2003 Workshop on Exploring the Trace Space for Dynamic Optimization Techniques. 51-58.

[16] Ian Hickson and David Hyatt. 2011. HTML5: a vocabulary and associated APIs for HTML and XHTML. W3C Working Draft, May 25, 2011.

[17] Paul Hsieh. 2004. Hash functions. Retrieved from http://www.azillionmonkeys. com/qed/hash.html.

[18] Urs Hölzle, Craig Chambers, and David Ungar. 1991. Optimizing dynamically-typed object-oriented languages with polymorphic inline caches. In Proceedings of the 1991 European Conference on Object-Oriented Programming (ECOOP'91). Springer-Verlag, London, 21-38.

[19] SungHyun Hong, Jin-Chul Kim, JinWoo Shin, Soo-Mook Moon, Hyeong-Seok Oh, Jaemok Lee, and Hyung-Kyu Choi. 2007. Java client ahead-of-time compiler for embedded systems. In Proceedings of the 2007 ACM SIGPLAN/SIGBED Conference on Languages, Compilers, and Tools for Embedded Systems (LCTES'07). ACM, New York, 63-72. DOI : http: //dx.doi.org/10.1145/1254766.1254776

[20] Pramod G. Joisha, Samuel P. Midkiff, Maurico J. Serrano, and Manish Gupta. 2001. A framework for efficient reuse of binary code in Java. In Proceedings of the 2001 15th International Conference on Supercomputing (ICS'01). ACM, New York, 440-453. DOI : http://dx.doi.org/10.1145/377792.377902

[21] Dong-Heon Jung, Soo-Mook Moon, and Sung-Hwan Bae. 2008. Design and optimization of a Java ahead-of-time compiler for embedded systems. In Proceedings of the 2008 IEEE/IFIP International Conference on Embedded and Ubiquitous Computing (EUC'08). IEEE, Washington, DC, 169-175. DOI : http://dx.doi.org/10.1109/EUC.2008.80

[22] Madhukar N. Kedlaya, Benham Robatmili and Ben Hardekopf. 2015. Server-side type profiling for optimizing clientside JavaScript engines. In Proceedings of the 2015 11th Symposium on Dynamic Languages (DLS 2015). New York, 140-153. DOI : http://dx.doi.org/10.1145/2816707.2816719

[23] Chandra J. Krintz, David Grove, Vivek Sarkar and Brad Calder. 2001. Reducing the overhead of dynamic compilation. Software: Practice and Experience 31, 8 (2001), 717-738. DOI : http://dx.doi.org/10.1002/spe.384

[24] Chris Marrin. 2011. WebGL specification. Khronos WebGL Working Group. 
[25] Mozilla. 2018. Firefox browser. Retrieved from https://www.mozilla.org/en-US/firefox/new.

[26] Mozilla. 2018. SpiderMonkey JavaScript engine. Retrieved from https://developer.mozilla.org/ko/docs/SpiderMonkey.

[27] Mozilla. 2018. asm.js. Retrieved from http://asmjs.org/.

[28] Node.js Foundation. 2018. Node.js. Retrieved from https://nodejs.org.

[29] Hyeong-Seok Oh, Ji Hwan Yeo, and Soo-Mook Moon. 2015. Bytecode-to-C ahead-of-time compilation for Android Dalvik virtual machine. In Proceedings of the 2015 Design, Automation \& Test in Europe Conference \& Exhibition (DATE'15). ACM, 1048-1053.

[30] JinSeok Oh and Soo-Mook Moon. 2015. Snapshot-based loading-time acceleration for web applications. In Proceedings of the 2015 International Symposium on Code Generation and Optimization (CGO'15). IEEE, Washington, DC, 179-189. DOI : http://dx.doi.org/10.1109/CGO.2015.7054198

[31] HyukWoo Park, Wonki Jung, and Soo-Mook Moon. 2015. JavaScript ahead-of-time compilation for embedded web platform. In Proceedings of the 2015 13th IEEE Symposium on Embedded Systems for Real-time Multimedia (ESTIMedia'15). IEEE, 49-57. DOI : http://dx.doi.org/10.1109/ESTIMedia.2015.7351768

[32] HyukWoo Park, SungKook Kim, and Soo-Mook Moon. 2017. Advanced ahead-of-time compilation for javascript engine (Work-in-progress). In Proceedings of the 2017 International Conference on Compilers, Architectures and Synthesis for Embedded Systems (CASES '17). ACM, New York, Article 16, 2 pages. DOI : http://dx.doi.org/10.1145/3125501. 3125512

[33] R. Radhakrishnan, J. Rubio, and L. K. John. 1999. Characterization of Java applications at bytecode and ultra-SPARC machine code levels. In Proceedings of the 1999 International Conference on Computer Design. IEEE, 108-115. DOI : http: //dx.doi.org/10.1109/ICCD.1999.808439

[34] R. Radhakrishnan, N. Vijaykrishnan, L. K. John, and A. Sivasubramaniam. 2000. Architectural issues in Java runtime systems. In Proceedings of the 2000 6th International Symposium on High Performance Computer Architecture (HPCA'00) IEEE, Touluse, France, 387-398. DOI : http://dx.doi.org/10.1109/HPCA.2000.824367

[35] R. Radhakrishnan, R. Bhargava, and L. K. John. 2001. Improving Java performance using hardware translation. In Proceedings of the 2001 15th International Conference on Supercomputing (ICS'01). ACM, New York, 427-439. DOI : http: //dx.doi.org/10.1145/377792.377901

[36] Paruj Ratanaworabhan, Benjamin Livshits, and Benjamin G. Zorn. 2010. JSMeter: comparing the behavior of JavaScript benchmarks with real web applications. In Proceedings of the 2010 USENIX conference on Web application development (WebApps'10). USENIX, Berkeley, CA, 3.

[37] Vijay Janapa Reddi, Dan Connors, Robert Cohn, and Michael D. Smith. 2007. Persistent code caching: exploiting code reuse across executions and applications. In Proceedings of the 2007 International Symposium on Code Generation and Optimization (CGO'07). IEEE, Washington, DC, 74-88. DOI : http://dx.doi.org/10.1109/CGO.2007.29

[38] Gregor Richards, Sylvain Lebresne, Brian Burg, and Jan Vitek. 2010. An analysis of the dynamic behavior of JavaScript programs. In Proceedings of the 2010 31st ACM SIGPLAN Conference on Programming Language Design and Implementation (PLDI'10). ACM, New York. 1-12. DOI : http://dx.doi.org/10.1145/1806596.1806598

[39] Mauricio Serrano, Rajesh Bordawekar, Sam Midkiff, and Manish Gupta. 2000. Quicksilver: a quasi-static compiler for Java. In Proceedings of the 2000 15th ACM SIGPLAN Conference on Object-Oriented Programming, Systems, Languages, and Applications (OOPSLA'00). ACM, New York, 66-82. DOI : http://dx.doi.org/10.1145/353171.353176

[40] Alon Zakai. 2011. Emscripten: an LLVM-to-JavaScript compiler. In Proceedings of the 2011 ACM International Conference Companion on Object Oriented Programming Systems Languages and Applications Companion (OOPSLA'11). ACM, New York, 301-312. DOI : http://dx.doi.org/10.1145/2048147.2048224

[41] R. Zhuykov and E. Sharygin. 2017. Ahead-of-time compilation of JavaScript programs. Programming and Computing Software 43, 1 (2017), 51-59. DOI : http://dx.doi.org/10.1134/S036176881701008X

Received April 2018; revised August 2018; accepted October 2018 